\title{
3D Atom Probe Microscopy Sample Preparation by Using L-Shape FIB-SEM-Ar Triple Beam
}

Xin Man, Tatsuya Asahata, Atsushi Uemoto, Hidekazu Susuki, Hiroyuki Suzuki, Masakatsu Hasuda and Toshiaki Fujii

Beam Technology Engineering Department, Hitachi High-Tech Science Corporation, Oyama-cho, Sunto-gun, Shizuoka, Japan.

$3 \mathrm{D}$ atom probe microscopy (3DAPM) is used as the technique of analyzing the three-dimensional information including chemical composition and atomic structure with near atomic-scale resolution [1]. The near atomic-scale resolution is realized by extracting atoms on a sample surface one by one with electric field evaporation. The needle-like sample with a tip diameter of about $50 \mathrm{~nm}$ is required in order to apply the local high electric field to the sample surface.

FIB-SEM is widely used for sample preparation for 3DAPM at a specific site [2]. Sample preparation is carried out by the following steps. i) Extracting a piece of a sample of the particular area containing the analysis object from a bulk sample by in-situ lift-out method. ii) Specifying the analysis object by SEM observation. iii) Sharpening the needle tip by FIB processing. The most important point in this sample preparation process is to make a needle-like sample with less than $50 \mathrm{~nm}$ of tip's diameter containing the analysis object. In the needle-like sample, it is very difficult to progress the sharpening of the sample without missing the feature of the analysis object which appears as contrast information on a SEM image. We developed the L-shape FIB-SEM-Ar triple beam which realizes the following functions to solve this subject, and it applied to sample preparation for 3DAPM.

1) During FIB/Ar ion beam processing, various information including chemical composition, crystal orientation and a transmission electron image acquired at beam coincident point can be utilized in addition to a SEM observation image for process end point detection.

2) SEM observation performance at beam coincident point is highly improved for specifying the small structure and a weak contrast pattern.

The features of the developed L-shape FIB-SEM-Ar triple beam are introduced. It is the main feature that the SEM column and the FIB column are arranged right-angled and this equipment optimizes arrangement of all detectors including EDS, EBSD, and STEM and Ar ion beam column to beam coincident point as it is shown in Figure 1. Thereby, this equipment makes it possible to obtain the information on all the detectors in real time during FIB/Ar ion beam processing. The sample stage is a five-axis eucentric stage with low drift and high precision positioning, and the axis of rotation is coincident with the direction of the FIB incidence. In addition, by limiting specimen size, WD of the SEM column at beam coincident point is shortened than standard FIB-SEM equipment, and then the observation performance by SEM at beam coincident point is highly improved.

The result of sample preparation for 3DAPM which targeted the specific grain boundary of the polycrystalline sample is shown in Figure 2. The crystal orientation map by EBSD and the low acceleration voltage SEM image acquired for the same sample surface of the pure iron is shown. The grain boundary between (111) plane and (101) plane was targeted in the state of 1.5-micrometer-wide quadrangular prism form. The position of the targeted grain boundary was checked in both the SEM 
image and the crystal orientation map by EBSD while sharpening the sample. The channeling contrast in SEM image decreased in the state where the diameter of the tip became $200 \mathrm{~nm}$ or less, and discernment of the grain boundary became difficult. On the other hand, on the crystal orientation map, the grain boundary has been identified in the state where it was sharpened to about $50 \mathrm{~nm}$ in diameter of the tip's apex. Thus, sample preparation containing specific grain boundary in the tip's apex has been realized by the ability of information other than SEM image to be simultaneously used in real time by L-shape FIBSEM-Ar Triple Beam. Moreover, in this system, the tip of the needle-like sample is processed with Ar ion beam, as rotating the needle-like sample on its axis to reduce the damage layer induced by Ga ion irradiation.

[1] D.J.Larson et al, Ultramicroscopy 79 (1999) p.287.

[2] W.R.McKenzie et al, Microscopy: Science, Technology, Application and Education Contents 3 (2010) p.1800

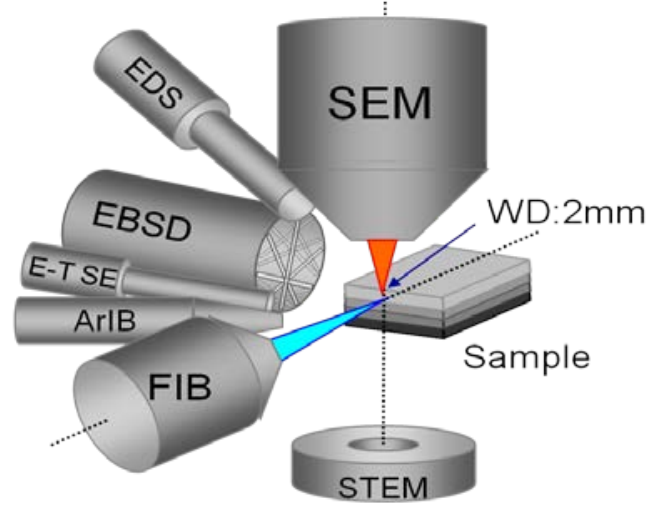

Figure 1. Unit Configuration of L-Shape FIB-SEM-Ar Triple Beam

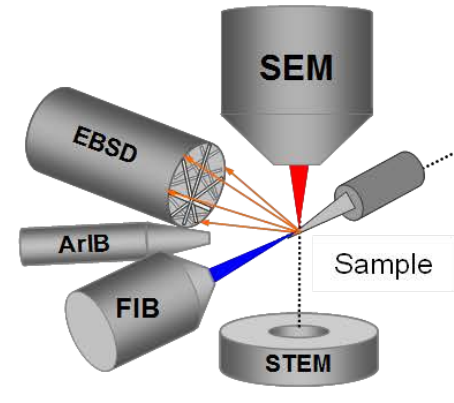

Iron - Alpha

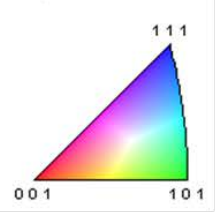

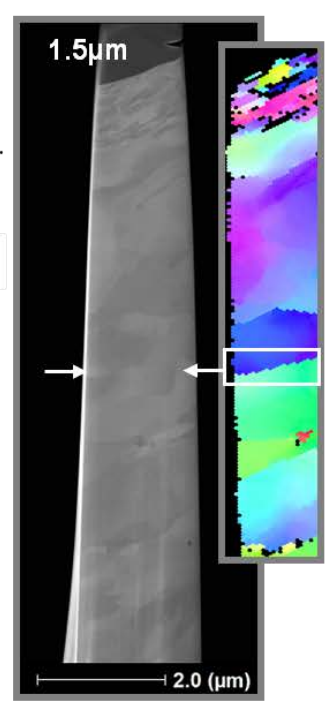
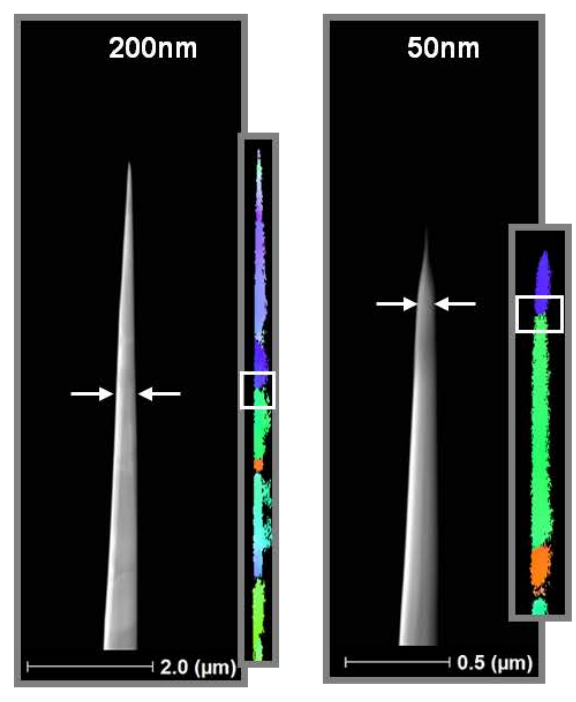

Figure 2. SEM image and crystal orientation map by EBSD for pure iron during sharpening. 